\title{
Galaxy lacking dark matter in the standard cosmological paradigm
}

\section{Jorge Moreno ( $\sim$ jorge.moreno@pomona.edu )}

Department of Physics and Astronomy, Pomona College https://orcid.org/0000-0002-3430-3232

\section{Shany Danieli}

Institute for Advanced Study

\section{James Bullock}

UC Irvine

\section{Robert Feldmann}

University of Zurich https://orcid.org/0000-0002-1109-1919

\section{Philip Hopkins}

Caltech

\section{Onur Catmabacak}

Institute for Computational Science, University of Zurich

\section{Alexander Gurvich}

Department of Physics \& Astronomy and CIERA, Northwestern University

\section{Alexandres Lazar}

University of California, Department of Physics and Astronomy, University of California, Irvine

\section{Courtney Klein}

Department of Physics and Astronomy, University of California, Irvine

\section{Cameron Hummels}

TAPIR, California Institute of Technology

\section{Zachary Hafen}

Department of Physics and Astronomy, University of California, Irvine

\section{Francisco Mercado}

Department of Physics and Astronomy, University of California, Irvine

\section{Sijie Yu}

Department of Physics and Astronomy, University of California, Irvine

\section{Fangzhou Jiang}

TAPIR, California Institute of Technology

\section{Coral Wheeler}

Department of Physics and Astronomy, California State Polytechnic University, Pomona

\section{Andrew Wetzel}

Department of Physics and Astronomy, University of California, Davis

\section{Daniel Angles-Alcazar}


Department of Physics, University of Connecticut

\section{Michael Boylan-Kolchin}

University of Texas at Austin https://orcid.org/0000-0002-9604-343X

\section{Quataert Eliot}

Department of Astrophysical Sciences, Princeton University

\section{Claude-Andre Faucher-Giguere}

Northwestern University https://orcid.org/0000-0002-4900-6628

\section{Dusan Keres}

UCSD

\section{Physical Sciences - Article}

Keywords: dark matter, galaxies, cosmology

Posted Date: August 12th, 2021

DOl: https://doi.org/10.21203/rs.3.rs-778732/v1

License: (c) (1) This work is licensed under a Creative Commons Attribution 4.0 International License. Read Full License

Version of Record: A version of this preprint was published at Nature Astronomy on February 14th, 2022. See the published version at https://doi.org/10.1038/s41550-021-01598-4. 


\section{Galaxies lacking dark matter in the standard cosmological paradigm}

3 Jorge Moreno*,1,2,3, Shany Danieli ${ }^{4}$, James S. Bullock ${ }^{2}$, Robert Feldmann ${ }^{5}$, Philip F. Hopkins ${ }^{3}$,

4 Onur Çatmabacak ${ }^{5}$, Alexander Gurvich $^{6}$, Alexandres Lazar $^{2}$, Courtney Klein $^{2}$, Cameron B. Hummels $^{3}$,

5 Zachary Hafen ${ }^{2}$, Francisco J. Mercado ${ }^{2}$, Sijie $\mathrm{Yu}^{2}$, Fangzhou Jiang ${ }^{3,7}$, Coral Wheeler ${ }^{8}$, Andrew

6 Wetzel $^{9}$, Daniel Anglés-Alcázar ${ }^{10,11}$, Michael Boylan-Kolchin ${ }^{12}$, Eliot Quataert ${ }^{13}$, Claude-André

7 Faucher-Giguère ${ }^{6}$ \& Dušan Kereš ${ }^{14}$

$8{ }^{1}$ Department of Physics and Astronomy, Pomona College, Claremont, CA 91711, USA

$9{ }^{2}$ Department of Physics and Astronomy, 4129 Reines Hall, University of California, Irvine, CA

92697, USA

${ }^{3}$ TAPIR, California Institute of Technology, Pasadena, CA 91125, USA

${ }^{4}$ Institute for Advanced Study, 1 Einstein Drive, Princeton, NJ 08540, USA

${ }^{5}$ Institute for Computational Science, University of Zurich, Winterthurerstrasse 190, Zurich CH8057, Switzerland

${ }^{6}$ Department of Physics \& Astronomy and CIERA, Northwestern University, 1800 Sherman Ave, Evanston, IL 60201, USA

${ }^{7}$ Carnegie Observatories, 813 Santa Barbara Street, Pasadena, CA 91101, USA

${ }^{8}$ Department of Physics and Astronomy, California State Polytechnic University, Pomona, Pomona, CA 91768, USA

${ }^{9}$ Department of Physics \& Astronomy, University of California, Davis, One Shields Ave, Davis, CA 95616 
${ }^{10}$ Department of Physics, University of Connecticut, 196 Auditorium Road, U-3046, Storrs, CT 06269-3046, USA

${ }^{11}$ Center for Computational Astrophysics, Flatiron Institute, 162 th Ave, New York, NY 10010, USA

${ }^{12}$ Department of Astronomy, The University of Texas at Austin, 2515 Speedway, Stop C1400, Austin, TX 78712-1205, USA

${ }^{13}$ Department of Astrophysical Sciences, Princeton University, Princeton, NJ 08544, USA

${ }^{14}$ Department of Physics and Center for Astrophysics and Space Sciences, University of California San Diego, 9500 Gilman Drive, La Jolla, CA 92093, USA

The standard cold dark matter plus cosmological constant model predicts that galaxies form within dark matter halos ${ }^{1}$ and that low-mass galaxies are more dark-matter dominated than massive ones ${ }^{2}$. The unexpected discovery of two low-mass galaxies lacking dark matter ${ }^{3,4}$ immediately provoked concerns about the standard cosmology and ignited explorations of alternatives, including self-interacting dark matter and modified gravity ${ }^{5-11}$. Apprehension ${ }^{5,11}$ grew after several simulations ${ }^{5,12-21}$ using the conventional cosmology failed to adequately form numerical analogs. Here we show that the standard paradigm naturally produces galaxies lacking dark matter, with characteristics largely in agreement with observations. Using a state-of-the-art cosmological simulation and a meticulous galaxy-identification technique, we find that extreme close-encounters with massive neighbors can transform regular galaxies into dark-matter deficient ones. We predict that $\sim 30 \%$ of massive central galaxies (with at least $10^{11}$ solar masses in stars) harbor at least one dark-matter deficient satellite 
(with $10^{8}-10^{9}$ solar masses in stars). This distinctive class of galaxies opens an additional layer to our understanding of the role of interactions in shaping galactic properties. Future observations surveying galaxies in the aforementioned regime will provide a crucial test of this scenario.

For over half a century, cold dark matter has been a key ingredient in our understanding of galaxy formation ${ }^{22,23}$. In the low-mass regime, the standard cold dark matter paradigm ${ }^{1}$ predicts that galaxies should be more dark-matter dominated ${ }^{2}$. This is supported by observations in the nearby Universe ${ }^{24}$. For this reason, the detection of DF2 and DF4, two low-mass galaxies devoid of dark matter, was not anticipated ${ }^{3,4}$. This intriguing discovery immediately sparked several searches of numerical analogs in cosmological simulations ${ }^{5,12-21}$, with limited success. Unsurprisingly, the absence of dark-matter deficient galaxies in these simulations also raised doubts on the validity of the standard paradigm itself ${ }^{5,11}$. In this paper, we demonstrate that a novel cosmological hydrodynamical simulation (which presupposes this paradigm) naturally creates numerical versions of similar dark matter-deficient galaxies. This simulation utilizes the 'Feedback In Realistic Environments' (FIRE-2) physics model ${ }^{25}$, which successfully reproduces an array of galaxy properties $^{26}$. This run is state-of-the-art in its ability to resolve the internal structure of individual galaxies within a large cosmological environment. We point the reader to the Methods Section for details. By directly comparing with observations, we confirm that some of our simulated galaxies resemble DF2 and DF4 in arresting ways. This finding alleviates the aforementioned concerns and restricts the need for alternative explanations invoking new (non-standard) physics, such as self-interacting dark matter ${ }^{11}$ and modified gravity ${ }^{5-10}$. 

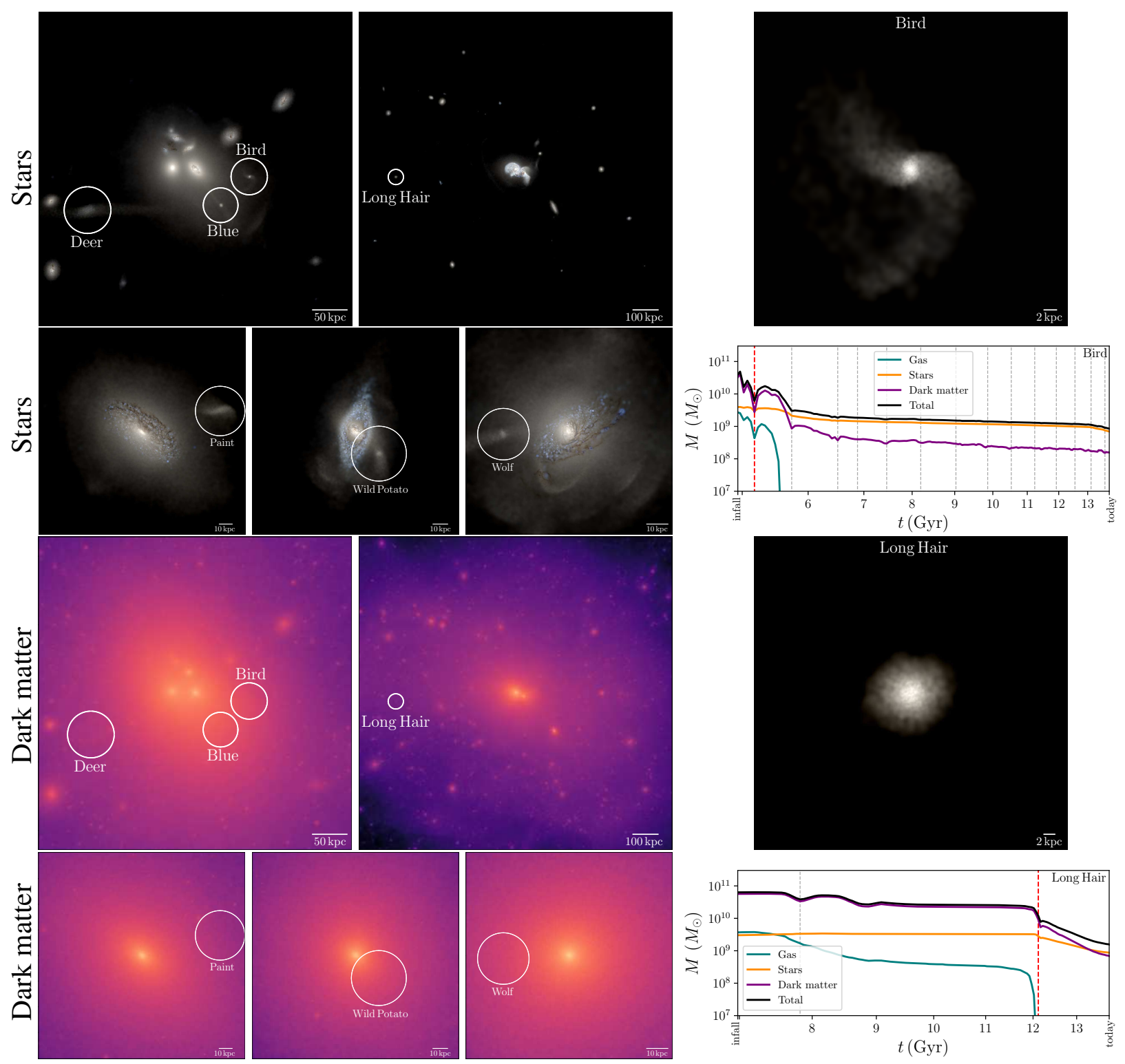

Figure 1: Galaxies lacking dark matter. Top left: Mock stellar Hubble Space Telescope $u / g / r$ composite images of the environments around our seven dark-matter deficient galaxies at present time $(z=0)$, limited to 29.5 mag $\operatorname{arcsec}^{-2}$. Bottom left: Dark matter mass surface density maps. The satellites are clearly visible in the stellar images but not in the dark matter maps. Right: Two examples - magnified mock stellar images and mass (inside the subhalo radius) versus time (teal, orange, purple and black represent gas, stars, dark matter and their sum). The vertical gray (red) lines depict (the closest) pericentric passages. 
We identify seven galaxies lacking dark matter within a volume of $10^{4} \mathrm{Mpc}^{3}$. Figure 1 shows present-time images of the environments around these simulated dark-matter deficient galaxies (white circles), which we name in honor of the seven Cherokee clans: Bird, Blue, Deer, Long Hair, Paint, Wild Potato and Wolf. The top-left panels show mock Hubble Space Telescope composite $u / g / r$ stellar images (down to $29.5 \mathrm{mag} \mathrm{arcsec}^{-2}$ ) and the bottom-left panels display corresponding dark matter mass surface density maps (down to $10^{5} M_{\odot} \mathrm{kpc}^{-2}$ ). The clear presence of these galaxies in the stellar images and not in the dark matter maps is remarkable. To illustrate, the right-hand panels display two special cases: Bird and Long Hair, the members with the most and least active interaction histories in our set of seven.

At the present time (redshift $z=0$ ), Bird, Blue and Deer inhabit the same galaxy group (virial mass $M_{\mathrm{vir}}=1.8 \times 10^{13} M_{\odot}$ ) and are at $80 \mathrm{kpc}, 66 \mathrm{kpc}$ and $174 \mathrm{kpc}$ away from its center, respectively. Long Hair also lives in a group $\left(M_{\mathrm{vir}}=1.4 \times 10^{13} M_{\odot}\right)$ and has the largest halocentric distance (484 kpc) amongst the seven. Paint, Wild Potato and Wolf exist in halos slightly more massive than that of the Milky Way $\left(M_{\mathrm{vir}}=3.6,2.5\right.$, and $\left.3.3 \times 10^{12} M_{\odot}\right)$ and have the following halo-centric distances: $72 \mathrm{kpc}, 57 \mathrm{kpc}$ and $36 \mathrm{kpc}$. The two observed galaxies lacking dark matter, $\mathrm{DF}^{3}$ and $\mathrm{DF}^{4}$, are at close projected separations $(80 \mathrm{kpc}$ and $165 \mathrm{kpc}$, which set a lower limit to the 3D separations) from NGC $1052^{27,28}$ (stellar mass $M_{\star}=1.05 \times 10^{11} M_{\odot}{ }^{29}$ ). Following Ref. ${ }^{30}$, we estimate that NGC 1052 has $M_{\text {vir }}=6.2_{-4.2}^{+25.8} \times 10^{12} M_{\odot}$ at $90 \%$ confidence, which is consistent with the virial masses of our hosts. Although halo-centric separations vary with time, their present-day values can provide information about their current membership status as satellites (see below). 


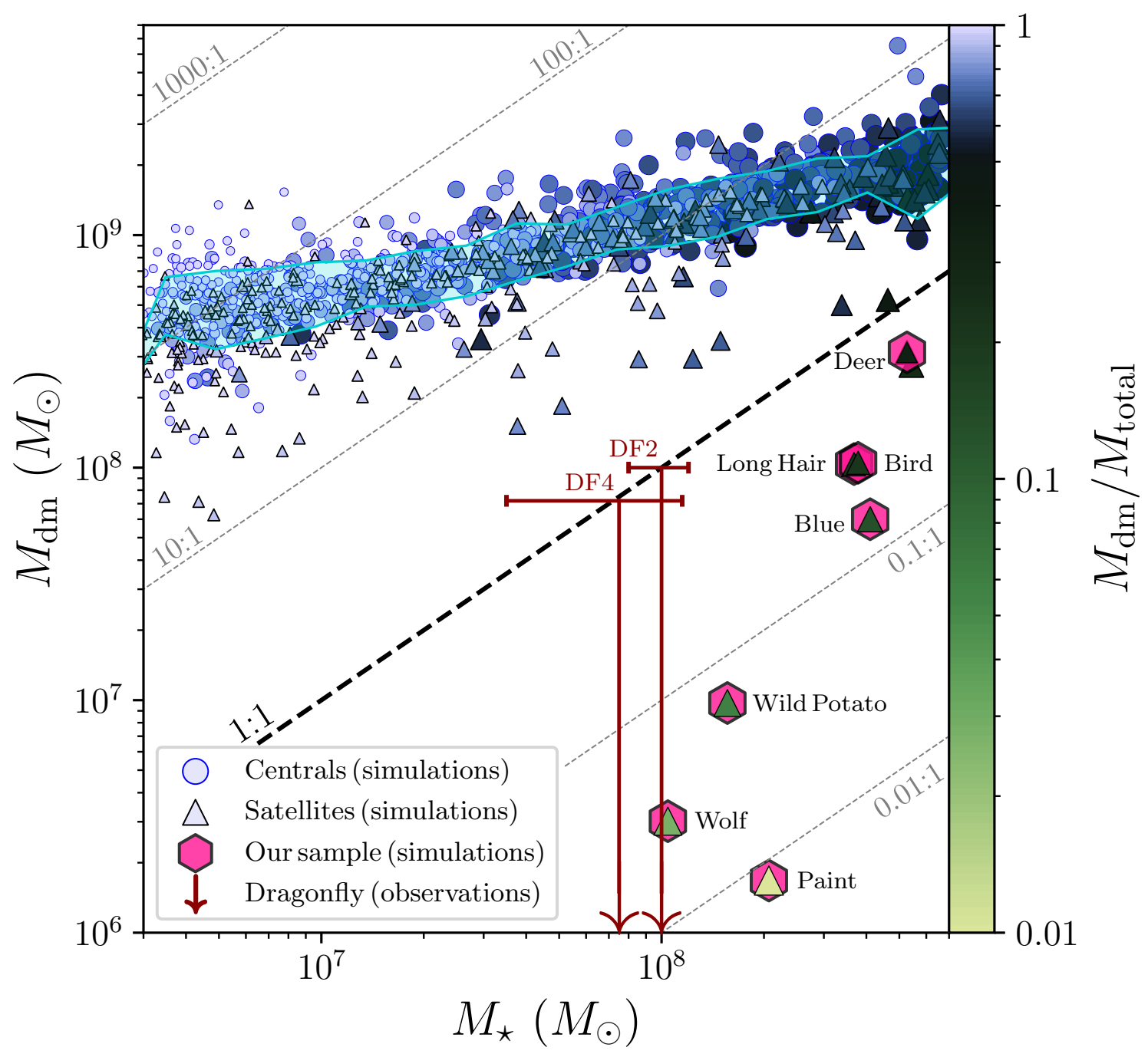

Figure 2: Fraction of mass in dark matter in simulated and observed galaxies. Dark matter mass versus stellar mass, color-coded by fraction of total mass in dark matter $\left(M_{\mathrm{dm}} / M_{\text {total }}\right)$. Masses are determined within $r_{50}^{\star}$, the radius containing $50 \%$ of the stellar mass. The solid circles and triangles denote central and satellites galaxies (simulations). Symbol dimensions scale inversely with $M_{\mathrm{dm}} / M_{\text {total }}$. The turquoise band represents the median and standard deviation (simulated centrals only). The pink hexagons highlight our sample of simulated galaxies lacking dark matter. The dark-red arrows with error bars represent Dragonfly observations. 
Figure 2 shows dark matter mass $\left(M_{\mathrm{dm}}\right)$ versus stellar mass $\left(M_{\star}\right)$. The diagonal lines represent constant dark-matter-to-stellar mass ratios. To compare with observations ${ }^{31}$ (dark-red arrows with error bars), hereafter we quote quantities within $r_{50}^{\star}$, the radius containing $50 \%$ of the stellar mass (we note, however, that our seven galaxies are also dark-matter deficient out to the subhalo radius). We select our sample of seven dark-matter deficient galaxies (pink hexagons) by requiring $M_{\mathrm{dm}}<M_{\star}$ (below the $1: 1$ line). Wolf is consistent with both DF2 and DF4 observations. We do not include galaxies with $M_{\star}<M_{\mathrm{dm}}<M_{\text {baryon }}$ in this sample. These are likely simulated analogs of the recently-discovered baryon-rich galaxies ${ }^{32,33}$, which we plan to study separately.

Figure 3 shows the 1D line-of-sight velocity dispersion $\left(\sigma_{\star}^{1 \mathrm{D}}\right)$ versus the galactic size $\left(r_{50}^{\star}\right)$. Hereafter, we only focus on satellites with $M_{\star}=10^{8-9} M_{\odot}$, in line with our dark-matter deficient sample. For observations of DF2 and DF4, we use deprojected measurements ${ }^{34}$ by Ref. ${ }^{31}$ and Ref. ${ }^{4}$, respectively. To guide the eye, the dashed diagonal lines track fixed dynamical masses within $r_{50}^{\star}$ according to an analytic mass estimator ${ }^{34}$. Our results show that Wolf and Wild Potato are consistent with DF4 and DF2, respectively. Additionally, to quantify the morphology of our seven galaxies, we performed Sérsic ${ }^{35}$ profile fittings in the $g$-band. We measure effective radii $\left(R_{\mathrm{e}}^{2 \mathrm{D}}\right)$, which are in line with our $r_{50}^{\star}$ values after deprojection (see the Methods Section). With this procedure, we also obtain Sérsic indices $\left(n_{\text {Sérsic }}\right)$, which range from $0.60-0.77$, in excellent agreement with observations ( $n_{\text {Sérsic }}=0.60$ and 0.79 for DF2 and DF4, respectively).

Previous cosmological simulations have not been able to match the internal properties of the two observed dark-matter deficient galaxies. They have also not been able to achieve dark-matter 


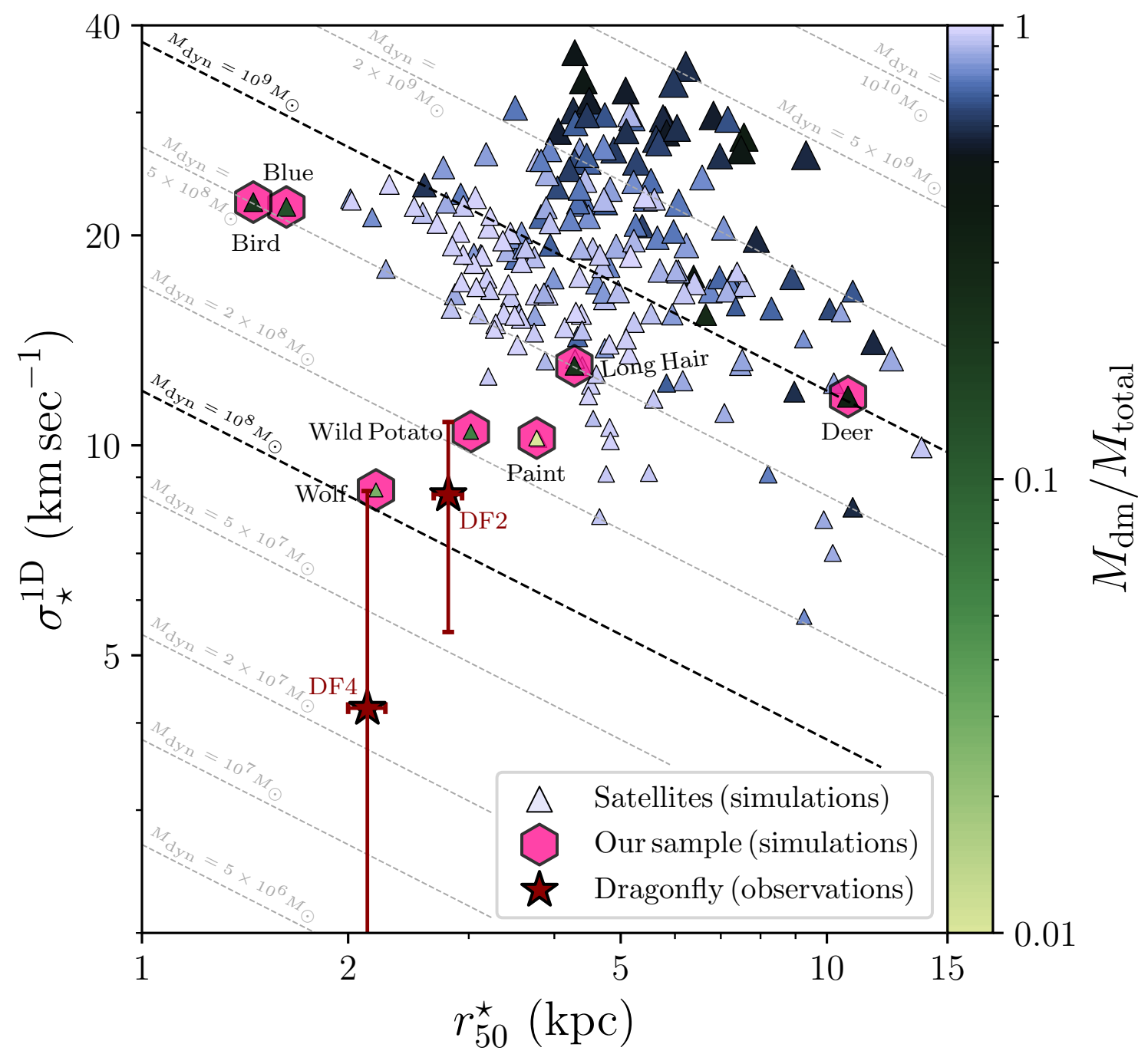

Figure 3: Comparison of internal properties between simulated and observed galaxies. Quantities are measured within $r_{50}^{\star}$. The triangles denote simulated satellites with $M_{\star}=10^{8-9} M_{\odot}$, their dimensions scale with $M_{\text {total }}$ and are color-coded by $M_{\mathrm{dm}} / M_{\text {total }}$. The dark-red stars with error bars represent Dragonfly observations ${ }^{4,31}$. The diagonal dashed lines show various fixed dynamical masses according to an analytic mass estimator ${ }^{34}: M_{\mathrm{dyn}}\left(<r_{50}^{\star}\right) \propto\left(\sigma_{\star}^{1 \mathrm{D}}\right)^{2} r_{50}^{\star}$. 
mass fractions as low as ours: three members of our sample have dark-matter mass fractions below $10 \%$ and one has only $1 \%$ of its mass in dark matter - rendering them almost dark-matter free galaxies. See the Methods Section for work by other groups ${ }^{5,12-21}$. One possible explanation is that our simulation has the distinct advantage in being able to model the small-scale $(\sim 20 \mathrm{pc})$ interstellar medium of individual galaxies within a cosmological region $(\sim 20 \mathrm{Mpc})$ large enough to contain several massive groups of galaxies.

Below we show that close encounters are responsible for the creation of dark-matter deficient galaxies in our simulation. Observationally, it is challenging to infer if a galaxy has experienced close interactions in its history. For instance, Bird exhibits 'S-shaped' low surface brightness tails, whilst Long Hair appears undisturbed (see magnified examples in Figure 1, right-hand side). However, our simulation reveals that close encounters with a massive neighbor are responsible for dark-matter deficiency in all of our seven galaxies. They all started out more massive, gas rich and with fairly typical stellar-to-dark-matter mass ratios for galaxies of their mass - but subsequently experienced multiple close interactions with their host galaxies (see mass-evolution insets in Figure 1 and the Methods Section). With the exception of Deer, these galaxies lost all of their gas during this process, in agreement with $\mathrm{DF}^{36}$. These satellites also lost between $97.9-99.99 \%$ of their dark matter mass, whilst losing only $45-97 \%$ of their stellar mass (within the subhalo radius). We suspect that stars are more resilient, as they move on more circular orbits in contrast to the eccentric orbits of the dark matter particles. The elongated orbits followed by the dark matter particles thus leave them more susceptible to tidal stripping ${ }^{37}$. We plan to verify this in future work. 


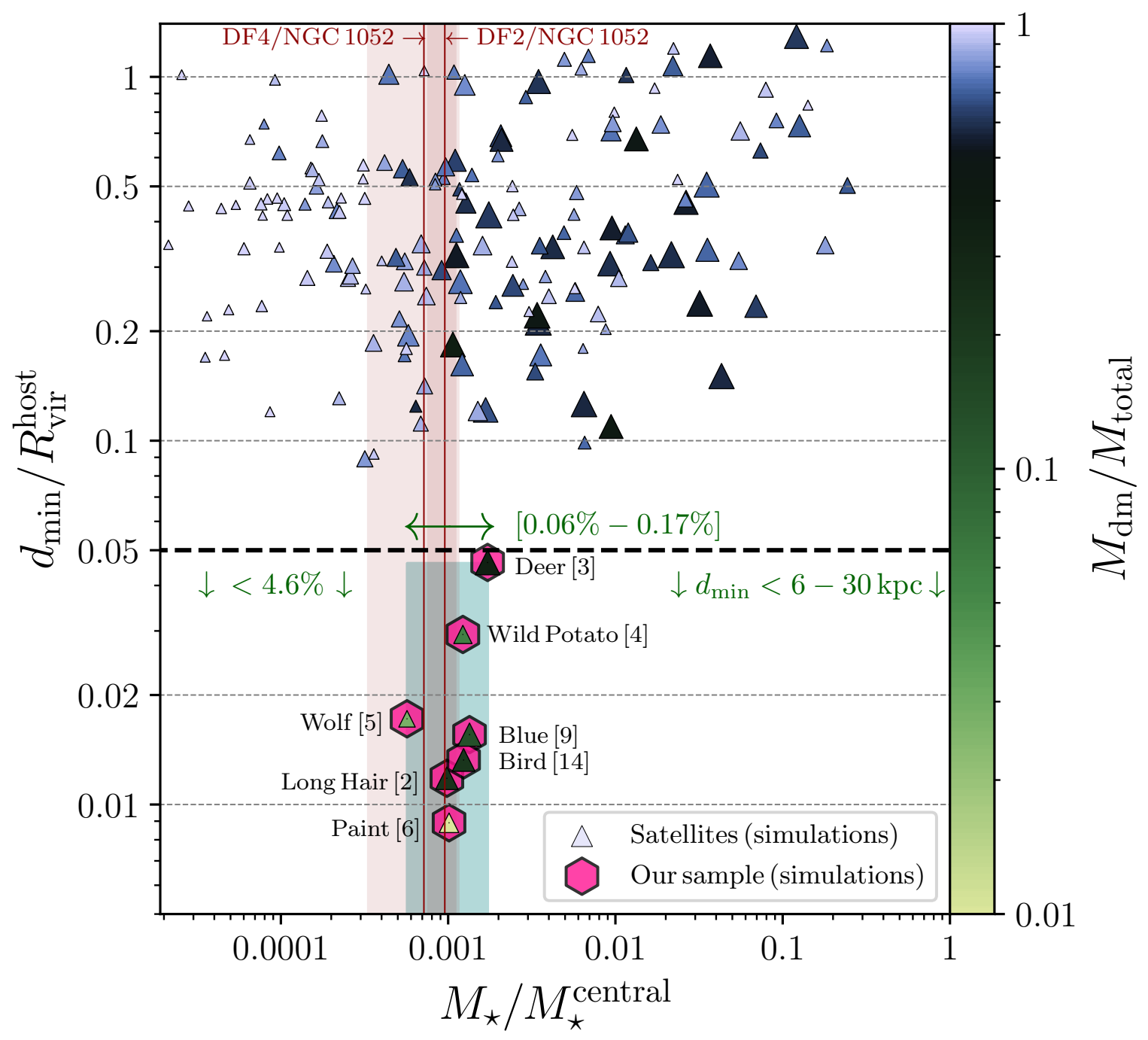

Figure 4: Conditions for creating a galaxy lacking dark matter. The minimum halo-centric distance ever achieved (in units of the hosts' virial radius) versus the satellite-central stellar mass ratio. The triangles denote simulated satellites with $M_{\star}=10^{8-9} M_{\odot}$, color coded by $M_{\mathrm{dm}} / M_{\mathrm{total}}$, with dimensions scaled by $M_{\star}$ (simulations). Quantities are within $r_{50}^{\star}$ for satellites and $r_{80}^{\star}$ for central companions. The turquoise rectangle and percentages highlight the region of parameter space our seven galaxies occupy and the numbers in brackets indicate number of pericentric passages. The vertical red lines (and bands) denote the stellar mass ratios (and uncertainty) between DF2/DF4 and NGC 1052 (observations). 
To quantify the role of interactions, we evaluate how close satellite galaxies come relative to their hosts and their stellar mass ratios relative to these massive central companions. Figure 4 shows $d_{\min } / R_{\text {vir }}^{\text {host }}$, the minimum halo-centric distance ever attained by each satellite (in units of the final virial radius of the host) versus $M_{\star} / M_{\star}^{\text {central }}$, the present-day satellite-central stellar-mass ratio. For satellites, we continue to employ $r_{50}^{\star}$, whilst for centrals, we switch to $r_{80}^{\star}$, which better captures their spatial extent ${ }^{38}$. Our simulation suggests that, to become a dark-matter deficient galaxy, a satellite must pierce within $\sim 5 \%$ (below horizontal black dashed line) of the host virial radius (within $\sim 6-30 \mathrm{kpc}$, depending on the host). In other words, the satellite must transit through the host's stellar body. We also predict that their satellite-central stellar-mass ratio must be $\sim 0.1 \%$. The stellar mass ratios of DF2 and DF4 relative to NGC 1052 are consistent with our simulation; however, no orbital information is available to determine their minimum halo-centric distance.

Whether or not the galaxies in our simulation were formed in the same way as DF2 and DF4 is still unclear. Our results support a scenario where DF2 and DF4 became dark-matter deficient due to past close interactions with NGC 1052, the only massive galaxy in their neighborhood (note that our simulation allows hosts to have multiple dark-matter deficient satellites). Their projected $(80 \mathrm{kpc}$ and $165 \mathrm{kpc})$ and line-of-sight separations $(2.1 \pm 0.5 \mathrm{Mpc})^{27}-$ as well as our estimates of the virial diameter of the NGC 1052 halo - suggest that DF2 and DF4 could be satellites of the NGC 1052 group. Concretely, using Ref. ${ }^{30}$, we estimate this group to have a virial diameter of $0.7-1.7 \mathrm{Mpc}$ at $90 \%$ confidence, allowing for the possibility that NGC 1052 could encompass both objects. This possibility is amplified if either DF2 or DF4 are a 'backsplash' ${ }^{39}$ galaxy - i.e., 
a galaxy on an extremely radial orbit that carried it beyond the virial volume of NGC 1052 after having had a close interaction (we note that our simulated sample includes two recent backsplash galaxies: Long Hair and Deer). Future investment in characterizing accurate 3D separations between DF2 and DF4 relative to NGC 1052 will provide an important test of this interaction-based scenario.

Another outstanding issue is the existence of anomalous globular cluster populations ${ }^{40}$ in DF2 and DF4. The simulation presented in this work has insufficient resolution to explore this. Using the same physics model employed here, Refs. ${ }^{41,42}$ are capable of re-creating bound star clusters using zoom-in simulations at higher resolution than ours. We suspect that, at some point in their history, the early gas-rich progenitors of our dark-matter deficient galaxies met the requisite conditions to produce bound star clusters. In the future, we plan to conduct higher-resolution zoomin simulations of our sample and ask if there is a correlation between dark-matter deficiency and the presence of a peculiar globular cluster population - and why the stellar component (including globular clusters) in these satellites is more resilient to tidal stripping than the dark matter.

Our results demonstrate that dark-matter deficient galaxies can arise naturally within the standard cold-dark-matter based cosmological paradigm. We note that did not expect this to occur a priori (i.e., our simulation was not originally designed for this purpose). Although there certainly is still room for new physics beyond the standard paradigm, discriminating between alternative models will now rely on predicted differences between the expected properties of dark-matter deficient galaxies, rather than their mere existence ${ }^{5}$. 
In this work we predict that, to efficiently uncover more galaxies devoid of dark matter, observers should focus on satellites with $M_{\star}=10^{8-9} M_{\odot}$, near massive companions with $M_{\star} \geq$ $10^{11} M_{\odot}$. However, we cannot exclude a situation where a central-satellite pair outside this mass regime could have a sufficiently unorthodox orbital configuration to produce the same effect. Verifying how strict this regime is will require a larger simulation box or multiple distinct realizations of this simulation (with the same physics, but different initial conditions). More generally, to truly constrain the varied processes and underlying physics governing these galaxies, ambitious observational campaigns - aimed at enumerating and characterizing large samples of galaxies with unusual dark-matter properties - will be desirable in the years ahead.

1. White, S. D. M. \& Rees, M. J. Core condensation in heavy halos: a two-stage theory for galaxy formation and clustering. MNRAS 183, 341-358 (1978).

2. Behroozi, P. S., Wechsler, R. H. \& Conroy, C. The Average Star Formation Histories of Galaxies in Dark Matter Haloes from z=0-8. ApJ 770, 57 (2013).

3. van Dokkum, P. et al. A galaxy lacking dark matter, Nature 555, 7698, 629-632 (2018).

4. van Dokkum, P. et al. A Second Galaxy Missing Dark Matter in the NGC 1052 Group. ApJL 874, 1 (2019).

5. Haslbauer, M., Banik, I., Kroupa, P. \& Grishunin, K. The ultra-diffuse dwarf galaxies NGC 1052-DF2 and 1052-DF4 are in conflict with standard cosmology. MNRAS 489, 2634-2651 (2019). 
6. Kroupa, P. et al. Does the galaxy NGC1052-DF2 falsify Milgromian dynamics? Nature 561, E4 (2018).

7. Moffat, J. W. \& Toth, V. T. NGC 1052-DF2 and modified gravity (MOG) without dark matter. MNRAS 482, L1-L3 (2019).

8. Khalifeh A. R. \& Jimenez R. Dwarf galaxies without dark matter: constraints on modified gravity. MNRAS 501, 254-260 (2021).

9. Famaey, B., McGaugh, S. \& Milgrom M. MOND and the dynamics of NGC 1052-DF2. MNRAS 480, 473-476 (2018).

10. Haghi, H. et al. A new formulation of the external field effect in MOND and numerical simulations of ultra-diffuse dwarf galaxies - application to NGC 1052-DF2 and NGC 1052-DF4. MNRAS 487, 2442-2454 (2019).

11. Yang, D., Yu, H.-B. \& An, H. Self-Interacting Dark Matter and the Origin of Ultradiffuse Galaxies NGC1052-DF2 and -DF4. PRL 125, 11 (2020).

12. Yu, H., Ratra, B. \& Wang, F.-J. Dark matter deficient galaxies in the Illustris flat- $\Lambda$ CDM model structure formation simulation. ArXiv e-prints (2018).

13. Jing, Y., et al. Dark-matter-deficient galaxies in hydrodynamical simulations. MNRAS 488, 3298-3307 (2009).

14. Haslbauer, M., et al. Galaxies lacking dark matter in the Illustris simulation. $A \& A$ 626, A47 (2019). 
15. Carleton, T. et al. The Formation of Ultra Diffuse Galaxies in Cored Dark Matter Halos Through Tidal Stripping and Heating. MNRAS 485, 382-395 (2019).

16. Sales, L. V., et al. The formation of ultradiffuse galaxies in clusters. MNRAS 494, 2, 1848-1858 (2020).

17. Saulder, C., et al. Isolated dark-matter-deprived galaxies in hydrodynamical simulations: real objects or artefacts? MNRAS 491, 1278-1286 (2020).

18. Shin, E., et al. Dark Matter Deficient Galaxies Produced via High-velocity Galaxy Collisions in High-resolution Numerical Simulations. ApJ 899, 25 (2020)

19. Jackson, R. A., et al. Dark matter-deficient dwarf galaxies form via tidal stripping of dark matter in interactions with massive companions. MNRAS 502, 2, 1785-1796 (2021).

20. Applebaum, E., et al. Ultrafaint Dwarfs in a Milky Way Context: Introducing the Mint Condition DC Justice League Simulations. ApJ 909, 2, 96 (2021).

21. Wright, A. C. The formation of isolated ultradiffuse galaxies in ROMULUS25. MNRAS 502, 4, 5370-5389 (2021).

22. Zwicky, E. Die Rotverschiebung von extragalaktischen Nebeln. HPA 6, 110-127 (1933).

23. Rubin, B. C. \& Ford, W. K. Jr. Rotation of the Andromeda Nebula from a Spectroscopic Survey of Emission Regions. ApJ 159, 379 (1970). 
24. Tollerud, E. et al. From Galaxy Clusters to Ultra-Faint Dwarf Spheroidals: A Fundamental Curve Connecting Dispersion-supported Galaxies to Their Dark Matter Halos. ApJ 726, 2, 23 (2011).

25. Hopkins, P. F. et al. FIRE-2 simulations: physics versus numerics in galaxy formation. MNRAS 480, 1, 800-863 (2018).

26. Faucher-Giguère, C.-A. Recent progress in simulating galaxy formation from the largest to the smallest scales. Nat. Ast. 2, 368-373 (2018).

27. Shen, Z. el al. A Tip of the Red Giant Branch Distance of $22.1 \pm 1.2 \mathrm{Mpc}$ to the Dark Matter Deficient Galaxy NGC 1052-DF2 from 40 Orbits of Hubble Space Telescope Imaging. ApJL 914, 1, L12, 9 (2021).

28. Danieli, S. et al. A Tip of the Red Giant Branch Distance to the Dark Matter Deficient Galaxy NGC 1052-DF4 from Deep Hubble Space Telescope Data. ApJL 895, 1, L4, 8 (2020).

29. Forbes, D. A. et al. The SLUGGS Survey: stellar masses and effective radii of early-type galaxies from Spitzer Space Telescope $3.6 \mu \mathrm{m}$ imaging. MNRAS 464, 4611-4623 (2017).

30. Zahid, H. J., Sohn, J. \& Geller, M. J. Stellar Velocity Dispersion: Linking Quiescent Galaxies to Their Dark Matter Halos. ApJ 859, 96, 10 (2018).

31. Danieli, S. et al. Still Missing Dark Matter: KCWI High-resolution Stellar Kinematics of NGC1052-DF2. ApJL 874, 2, L12, 8 (2019). 
32. Mancera Piña, P. et al. Off the Baryonic Tully-Fisher Relation: A Population of Baryondominated Ultra-diffuse Galaxies ApJ 883, L33 (2019).

33. Guo, Q. et al. Further evidence for a population of dark-matter- deficient dwarf galaxies. Nat. Ast. 4, 246-251 (2020).

34. Wolf, J. et al. Accurate masses for dispersion-supported galaxies. MNRAS 406, 1220-1237 (2010).

35. Sérsic, J. L., Influence of the atmospheric and instrumental dispersion on the brightness distribution in a galaxy. BAAA 6, 41 (1963).

36. Sardone, A. et al. Constraints on the HI Mass of NGC 1052-DF2. ApJ 871, 2, 31, 5 (2019).

37. Peñarrubia, J. et al. The impact of dark matter cusps and cores on the satellite galaxy population around spiral galaxies. MNRAS 406, 1290-1305 (2010).

38. Mowla, L., van der Wel, A., van Dokkum, P. \& Miller, T. B. A Mass-dependent Slope of the Galaxy Size-Mass Relation out to $\mathrm{z} \sim 3$ : Further Evidence for a Direct Relation between Median Galaxy Size and Median Halo Mass. ApJL 872, L13 (2019).

39. Wetzel, A. R. et al. Galaxy evolution near groups and clusters: ejected satellites and the spatial extent of environmental quenching. MNRAS 439, 2687-2700 (2014).

40. Shen, Z. el al. A Complex Luminosity Function for the Anomalous Globular Clusters in NGC 1052-DF2 and NGC 1052-DF4. ApJ 909, 2, 179, 10 (2021). 
41. Kim, J. h. et al. Formation of globular cluster candidates in merging proto-galaxies at high redshift: a view from the FIRE cosmological simulations. MNRAS 474, 3, 4232-4244 (2018).

42. Ma, X., et al. Self-consistent proto-globular cluster formation in cosmological simulations of high-redshift galaxies. MNRAS 493, 3, 4315-4332 (2020).

\section{Methods}

Our cosmological simulation. We ran a high-resolution cosmological simulation of galaxy formation with box size of $21 \mathrm{cMpc}$ and the following number of baryonic and dark matter particles: $N_{\mathrm{b}}=1,024^{3}$ and $N_{\mathrm{DM}}=1,024^{3}$. Initially the baryonic particle masses are $m_{\mathrm{b}}=6.3 \times 10^{4} M_{\odot}$ for gas and star particles, whilst the dark matter particles have $m_{\mathrm{DM}}=3.3 \times 10^{5} M_{\odot}$. The force resolution is set at a fixed $h_{\text {star }}=12 \mathrm{pc}$ (physical) for star particles and $80 \mathrm{pc}$ for dark matter particles. For gas, the force resolution is set to equal the adaptive smoothing length down to a minimum of $1.5 \mathrm{pc}$, which occurs only in the densest regions of galaxies. We identify galaxies (and merger history trees) with the AMIGA Halo Finder $\left(\mathrm{AHF}^{1}\right)$, which uses an iterative unbinding procedure to identify gravitationally-bound objects ${ }^{2}$. We used $y t^{3}$ to interface with the particle data. The cosmological parameters are set to $\Omega_{\mathrm{m}}=0.3089, \Omega_{\Lambda}=0.6911, \Omega_{\mathrm{b}}=0.0486, \sigma_{8}=0.8159$ and $h=0.6774$.

We employ the 'Feedback In Realistic Environments' (FIRE-2) model ${ }^{25}$ for baryonic physics, which includes radiative cooling, star formation in dense self-gravitating gas and accounts for stellar feedback in the form of supernovae, stellar mass-loss and radiation interacting with the surrounding gas (as described therein). This model has been extensively validated in a number 
of publications analyzing properties of galaxies across a range in stellar masses and numerical resolutions ${ }^{26}$. In our run, star formation occurs only in gas denser than $n=300 \mathrm{~cm}^{-3}$. With this condition, the inter-particle separation in star-forming gas is $\sim 20 \mathrm{pc}$ (or less) and we are able to resolve giant-molecular-cloud complexes within the interstellar medium of individual galaxies.

Our simulated galaxy samples. In this work we focus on galaxies with at least 100 stellar particles at redshift $z=0$. The actual number of particles per object is typically much larger owing to an additional contribution of gas and dark matter particles for each object, either at the present time or at some point in its history. To avoid discarding galaxies lacking dark matter, our application of AHF utilizes all types of particles, not just dark matter. This comes at a price: the halo finder falsely identifies large numbers of gas clumps within galaxies as individual 'galactic units'. To address this, first we discard objects with baryon-to-total mass ratios greater than 0.5 . This may naturally discard the very objects we seek (and keep false clumps below this threshold). To avoid this, we create surface density maps of every halo with substructures and visually recover objects that might have been discarded erroneously by our $50 \%$ cut. The images shown in Figure 1 belong to this set. We focus on manually deleting clumps embedded inside massive galaxies and on recovering galaxies that are either clearly disjoint from their neighbors - or in tidal tails, to avoid rejecting tidally-formed candidates ${ }^{4}$. We highlight that every galaxy lacking dark matter studied in this paper is recovered during this step. Lastly, because we are interested in the low-mass regime, we constrain our sample to have stellar masses under $10^{9} M_{\odot}$ (within $r_{80}^{\star}$, the radius containing $80 \%$ of the stellar mass). This produces our final parent sample, which contains 1,218 resolved galaxies: 886 centrals and 332 satellites. 
Our dark-matter deficient set consists of seven galaxies with $M_{\mathrm{dm}}<M_{\star}$ that are not participating in a major merger (we discard an eighth galaxy meeting the former condition, but not the latter). We adopt this extra condition because the two observed Dragonfly galaxies are not currently merging with a companion of similar mass. We also exclude 19 low-mass galaxies (15 centrals and 4 satellites) with $M_{\mathrm{dm}}<M_{\text {baryon }}$ (but $M_{\mathrm{dm}}>M_{\star}$ ) - which are possibly numerical analogs of the recently-observed baryon-rich galaxies ${ }^{32,33}$. The final set of seven are satellites with $M_{\star}=10^{8-9} M_{\odot} ;$ they orbit five different massive central galaxies with $M_{\star}>10^{11} M_{\odot}$. In total, our simulation contains fifteen massive centrals that host 47 satellite galaxies in the target stellar mass range $\left(10^{8-9} M_{\odot}\right)$. Of these satellites, $\sim 15 \%$ belong to our set of seven, which orbit $\sim 30 \%$ of the available massive centrals.

The lack of dark-matter deficient satellite galaxies at stellar masses below $10^{8} M_{\odot}$ could arise from the fact that, at lower masses, galaxies are born as centrals with very high dark matter mass fractions, such that environmental effects cannot act to remove dark matter without completely destroying the galaxy. The absence of dark-matter deficient galaxies at lower stellar masses is likely not driven by lack of resolution because our simulation produces a large population of galaxies with at least 1,000 stellar particles that are less massive than Wolf (which has 3,280 stellar particles at redshift zero and had a factor of $\sim 35$ higher before becoming a satellite).

Galactic sizes. We calculate galactic sizes as follows. For each object, we record the distance to the nearest major companion (with stellar mass of at least a tenth of that of our object of interest). If no such companion exists, we record the virial radius (for centrals) or subhalo radius (for satellites). This step is necessary because AHF considers substructures as part of their host. Once this region 


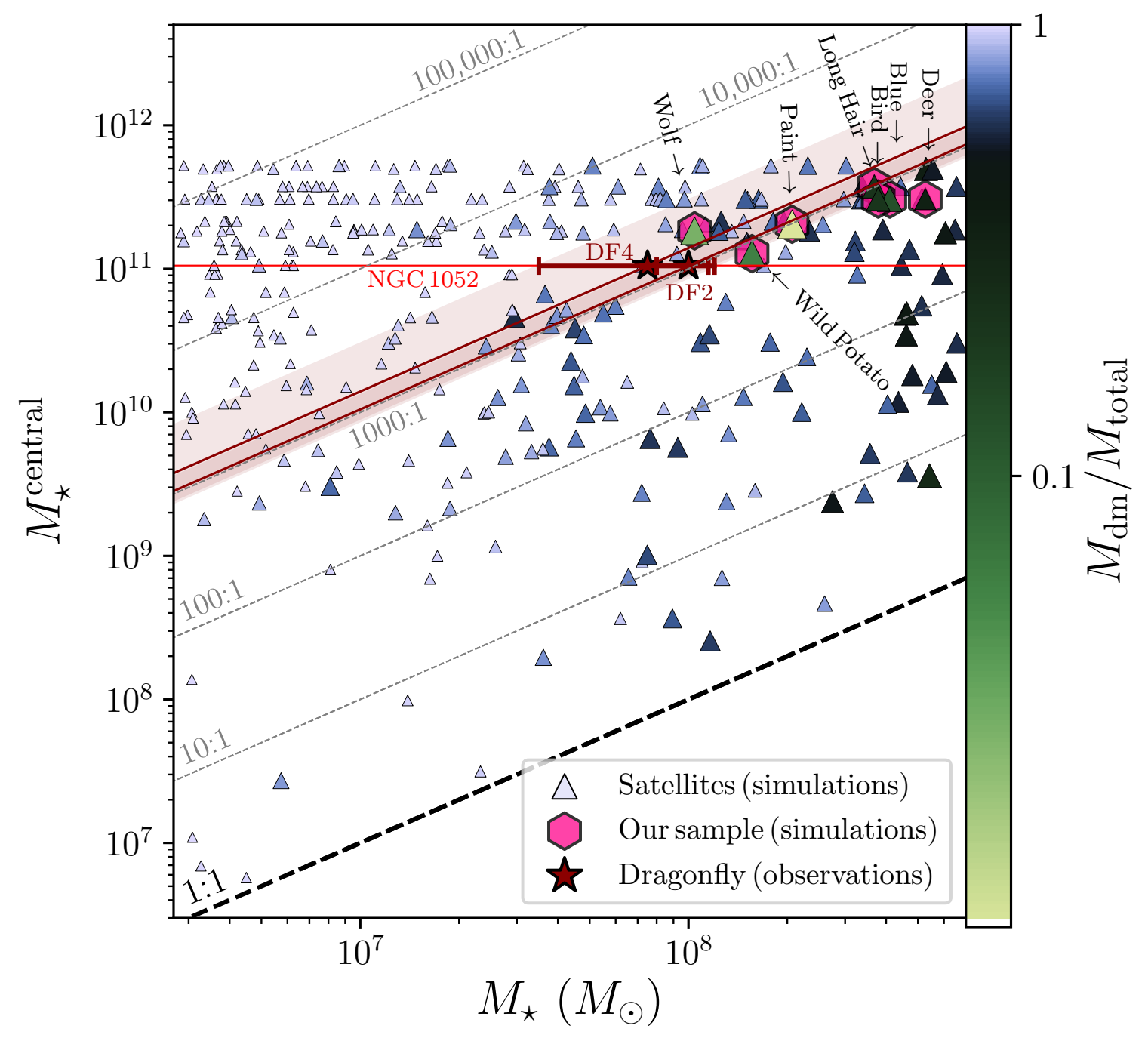

Figure 5: Stellar masses of satellite and central galaxies. We display the stellar mass of the central galaxy (within $r_{80}^{\star}$ ) versus that of each satellite (within $r_{50}^{\star}$ ). We adopt the same symbol and color scheme as in Figure 4. The horizontal red line refers to NGC 1052's stellar mass and the dark-red stars represent DF2 and DF4. The diagonal light-gray dashed lines indicate constant central-to-satellite stellar mass ratios. The brown diagonal lines (and bands) indicate the stellarmass ratios between DF2 or DF4 and NGC 1052 (and their uncertainties). 
is identified, we calculate the stellar center-of-mass. Next we redefine the radius of this region by subtracting the distance from the origin to this center-of-mass. Within this region, we then calculate cumulative mass profiles (using 200 identical linear radial bins) and extract the radius containing $80 \%$ of the stellar mass within this region by linearly interpolating between the two radii below and above this threshold.

$1 D$ line-of-sight velocity dispersions. We also calculate 1D line-of-sight velocity dispersions $\left(\sigma_{\star}^{1 \mathrm{D}}\right)$ within $r_{50}^{\star}$. We perform this calculation along three random directions (in the frame of each galaxy), corresponding to the $x, y$ and $z$ directions in the frame of the box. We create histograms of line-of-sight velocity minus the average line-of-sight velocity within the $r_{50}^{\star}$. These histograms have bin-widths of $2 \mathrm{~km} \mathrm{sec}^{-1}$ and extend from -70 to $70 \mathrm{~km} \mathrm{sec}^{-1}$. We then fit a Gaussian curve and record its $\sigma$-value. In the main text we report the simple average of these three numbers.

Host properties. Figure 5 shows the stellar mass of the central galaxies (hosting galaxies in our low-mass sample) versus that of their satellites. We employ $r_{80}^{\star}$ for the former (to better capture the spatial extent of the galaxy ${ }^{38}$ ) and $r_{50}^{\star}$ for the latter (for easy comparisons with observations). The diagonal lines denote constant stellar-mass ratios. Our seven dark-matter deficient galaxies, which have stellar masses between $10^{8-9} M_{\odot}$, accompany massive central galaxies in a narrow stellar-mass regime between $(1.3-3.7) \times 10^{11} M_{\odot}$. The stellar mass ratios of our seven galaxies are similar to that of DF2 and DF4 relative to NGC 1052 (diagonal brown lines and bands).

Characteristics of our dark-matter deficient sample. Figures $6 \mathrm{a}$ and $6 \mathrm{~b}$ display details for each member in our sample of simulated dark-matter deficient galaxies. The left column shows mock 

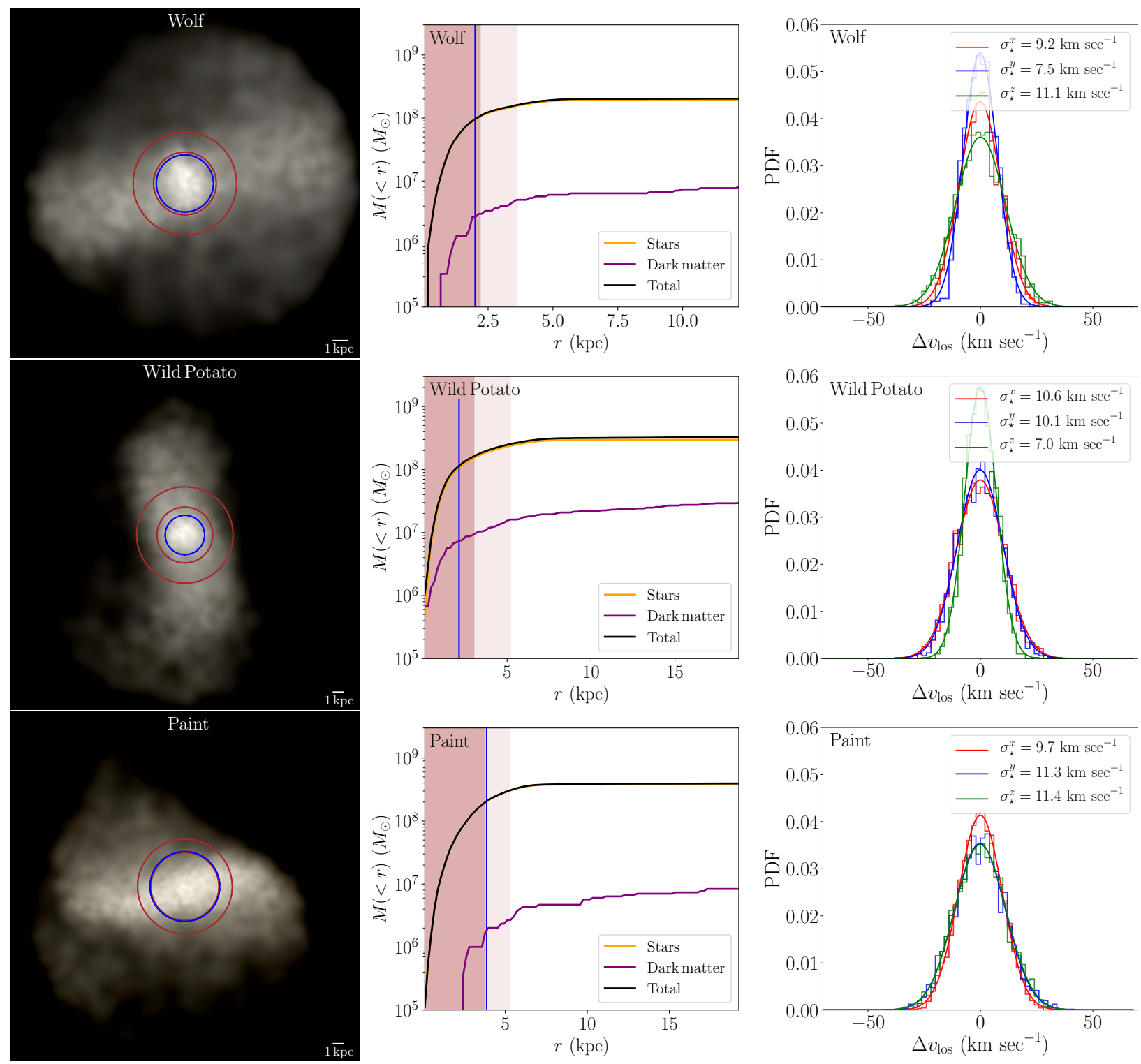

Figure 6a: Characteristics of the seven dark-matter deficient galaxies. Left column: Mock

Hubble Space Telescope $u / g / r$ composite stellar images (inner and outer red circles denote $r_{50}^{\star}$ and $r_{80}^{\star}$, blue circle represents $(4 / 3) R_{\mathrm{e}}^{2 \mathrm{D}}$ in the $g$-band; field-of-view equals the subhalo radius). Middle column: 3D cumulative mass profiles. Teal, orange and purple curves denote gas, stars and dark matter. Black represents their sum. The dark and light rectangles denote $r_{50}^{\star}$ and $r_{80}^{\star}$; the blue vertical line denotes $(4 / 3) R_{\mathrm{e}}^{2 \mathrm{D}}$ ( $g$-band). Right column: 1D line-of-sight stellar velocity dispersion within $r_{50}^{\star}$ along three random orthogonal directions ( $x, y$ and $z$ directions in the frame of the box). 

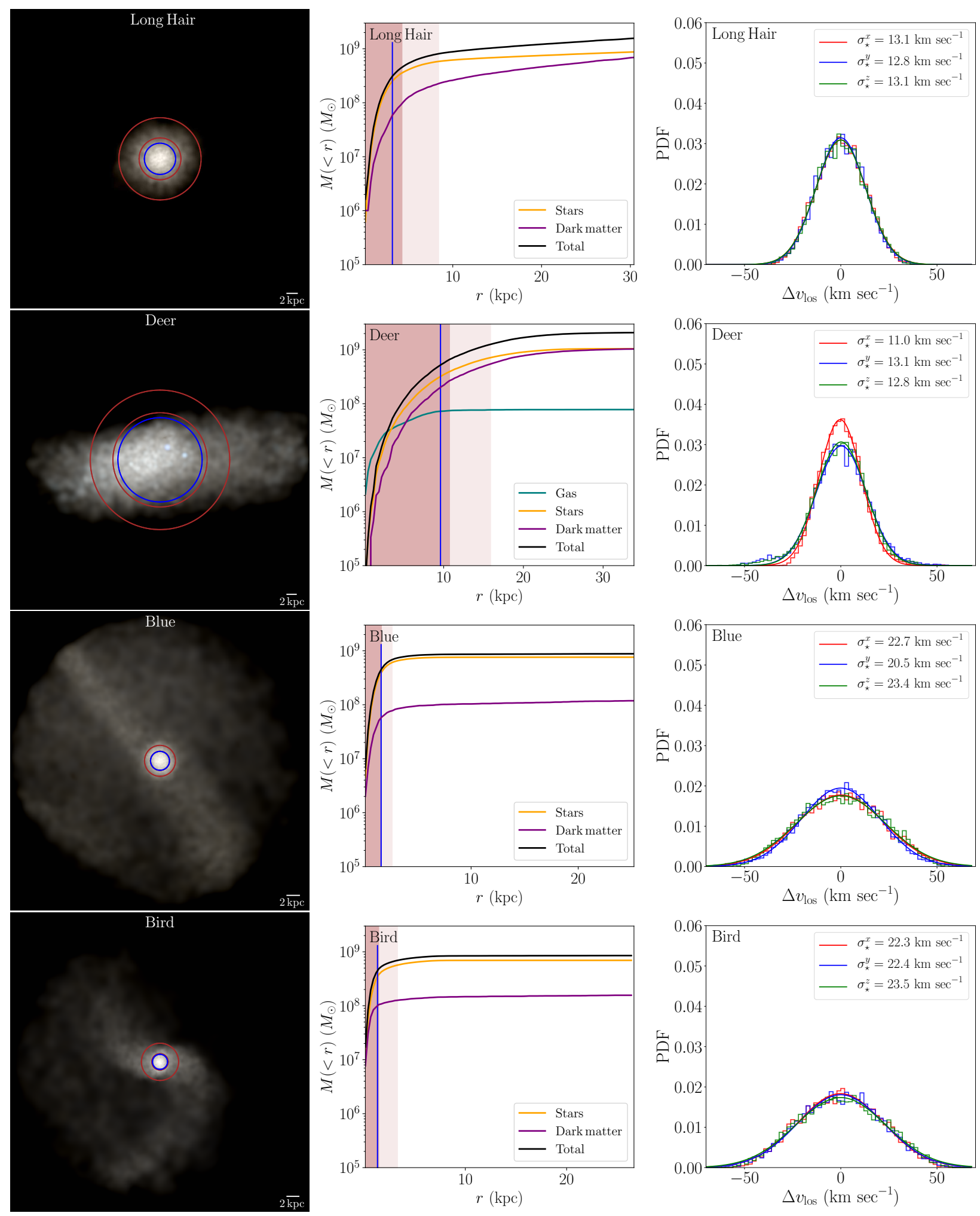

Figure 6b: Continuation of Figure 6a. 


\begin{tabular}{||c||ccccccc|cc||}
\hline & Bird & Blue & Deer & LH & Paint & WP & Wolf & DF2 & DF4 \\
\hline \hline$M_{\star}\left(10^{8} M_{\odot}\right)$ & 3.8 & 4.1 & 5.3 & 3.7 & 2.1 & 1.6 & 1.0 & $1.0 \pm 0.2$ & $0.75 \pm 0.4$ \\
\hline$M_{\text {gas }}\left(10^{8} M_{\odot}\right)$ & 0.0 & 0.0 & 0.5 & 0.0 & 0.0 & 0.0 & 0.0 & - & - \\
\hline$M_{\mathrm{dm}}\left(10^{8} M_{\odot}\right)$ & 1.0 & 0.6 & 3.1 & 1.0 & 0.02 & 0.1 & 0.03 & - & - \\
\hline$M_{\text {total }}\left(10^{8} M_{\odot}\right)$ & 4.8 & 0.6 & 8.9 & 4.7 & 2.1 & 1.7 & 1.1 & $1.3 \pm 0.8$ & - \\
\hline$\sigma_{\star}^{1 \mathrm{D}}\left(\mathrm{km} \mathrm{sec}^{-1}\right)$ & 22.3 & 22.0 & 11.7 & 13.0 & 10.2 & 10.5 & 8.6 & $8.5_{-3.1}^{+2.3}$ & $4.2_{-2.2}^{+4.4}$ \\
\hline$r_{50}^{\star}(\mathrm{kpc})$ & 1.5 & 1.6 & 10.7 & 4.3 & 3.8 & 3.0 & 2.2 & - & - \\
\hline$R_{\mathrm{e}}^{2 \mathrm{D}}(\mathrm{kpc})$ & 1.0 & 1.2 & 7.2 & 2.4 & 2.9 & 1.6 & 1.5 & $2.2 \pm 0.1$ & $1.6 \pm 0.1$ \\
\hline$n_{\text {Sérsic }}$ & 0.77 & 0.60 & 0.70 & 0.67 & 0.58 & 0.73 & 0.60 & 0.6 & 0.79 \\
\hline$M_{\star}^{\text {central }}\left(10^{11} M_{\odot}\right)$ & 3.1 & 3.1 & 3.1 & 3.7 & 2.0 & 1.3 & 1.8 & 1.0 & 1.0 \\
\hline$M_{\text {vir }}^{\text {host }}\left(10^{13} M_{\odot}\right)$ & 1.8 & 1.8 & 1.8 & 1.4 & 0.4 & 0.3 & 3.3 & $0.6_{-0.4}^{+2.6}$ & $0.6_{-0.4}^{+2.6}$ \\
\hline$d_{\text {host }}(\mathrm{kpc})$ & 80.2 & 66.3 & 174.1 & 483.8 & 72.3 & 56.9 & 36.2 & $>80$ & $>165$ \\
\hline$d_{\text {min }}(\mathrm{kpc})$ & 9.1 & 10.7 & 31.7 & 7.4 & 3.6 & 10.4 & 6.7 & - & - \\
\hline
\end{tabular}

Table 1: Characteristics of our dark-matter deficient galaxies. 'LH' and 'WP' denote Long Hair and Wild Potato. Masses are within $r_{50}^{\star}$, the radius containing 50\% of the stellar mass, unless stated otherwise. (i) Stellar mass; (ii) gas mass; (iii) dark matter mass; (iv) total mass; (v) 1D line-of-sight velocity dispersion; (vi) galactic size; (vii) the effective 2D radius ( $g$-band stellar light); (viii) Sérsic ${ }^{35}$ index; (ix) stellar mass of accompanying central within $r_{80}^{\star}$; (x) host's virial mass $\left(90 \%\right.$ confidence $\left.^{30}\right)$; (xi) present-time halo-centric distance (projected distance is used for observations); (xii) minimum halo-centric distance. 
Hubble space telescope $u / g / r$ composite stellar images (created with Fire Studio, an open source Python visualization package). We employ the same surface brightness limit as in Figure 1: $29.5 \mathrm{mag} \operatorname{arcsec}^{-2}$. The field of view corresponds to the subhalo radius. The inner and outer red circles denote $r_{50}^{\star}$ and $r_{80}^{\star}$, respectively. The blue circles denote the $g$-band effective radius ( $R_{\mathrm{e}}$, see below). The second column shows cumulative 3D-radial mass profiles within the subhalo radius. The dark and light vertical rectangles represent $r_{50}^{\star}$ and $r_{80}^{\star}$, respectively. The vertical blue line represents $R_{\mathrm{e}}$. The teal, orange, purple and black lines represent gas (when available), stars, dark matter and the sum of these (the total mass). The right column shows histograms and Gaussian best-fitting lines for 1D line-of-sight velocity dispersions with $r_{50}^{\star}$. We report values using particles within a sphere with this radius. Using a projected circular region yields very similar results. See Table 1 for a list of key properties.

With the exception of Long Hair, all of our simulated dark-matter deficient galaxies exhibit low surface brightness tidal features. Deer is the only member of our set with a detectable (albeit subdominant) gaseous component. For Wolf, Wild Potato and Paint, the stellar cumulative mass profiles dominate at all galactocentric radii (the orange and black curves are almost indistinguishable). This is particularly evident for Paint, the galaxy with the lowest dark-matter-to-total mass fraction in our simulation (the purple curve is $\sim 2$ dex below the orange curve at all radii). Wolf, Wild Potato and Paint are the only galaxies that achieve 1D line-of-sight velocity dispersions below $10 \mathrm{~km} \mathrm{sec}^{-1}$ - which has been a challenge to produce in cosmological simulations ${ }^{15,16}$. In addition to lacking low surface brightness features, Long Hair is also the galaxy with the largest halo-centric distance today. Note that its line-of-sight velocity dispersion histograms and curves 
along the three orthogonal directions are almost indistinguishable, suggesting that this galaxy has had enough time at distances far away from its host to become dynamically relaxed.

We recognize that it is observationally impossible to directly measure stellar-mass-based 3D sizes. To emulate what is often done, we also measure the effective radii $\left(R_{\mathrm{e}}^{2 \mathrm{D}}\right)$ of the seven darkmatter deficient galaxies by fitting a Sérsic ${ }^{35}$ profile to the average surface brightness in the $g$-band along each principal axis. In Figures $6 \mathrm{a}$ and $6 \mathrm{~b}$, we report the simple average of $R_{\mathrm{e}}^{2 \mathrm{D}}$ calculated along three (random) orthogonal directions (blue circles, using a deprojection factor ${ }^{34}$ of $4 / 3$ ) which agrees well with our 3D method. Table 1 includes these values, along with the associated Sérsic indices $\left(n_{\text {Sérsic }} \simeq 0.60-0.77\right)$, which are also in line with observations $\left(n_{\text {Sérsic }}=0.60\right.$ and 0.79 for DF2 and DF4, respectively).

The evolution of our dark-matter deficient sample. Figures $7 \mathrm{a}$ and $7 \mathrm{~b}$ show the evolution of our seven dark-matter deficient galaxies. The left panels show halo-centric distance (in physical units) versus cosmic time, between infall time (defined as the last time the galaxy was a central) and today. The solid and dotted gray lines represent the growth of the virial radius of the host and 5\% thereof. The thin vertical dashed gray lines indicate pericentric passages and the thick vertical red dashed line corresponds to the minimum halo-centric distance. We confirmed the aformentioned number of close passages by inspecting 3D representations of these orbits (not included). The right panels show mass (within the subhalo radius) versus time. The teal, orange, purple and black curves represent mass in gas, stars, dark matter and the sum of these, respectively. Before becoming satellites, our seven galaxies were more massive $\left(M_{\star} \sim 2-10 \times 10^{9} M_{\odot}\right)$, gas-rich $\left(M_{\text {gas }} \sim 3-25 \times 10^{9} M_{\odot}\right)$ and dark-matter dominated $\left(M_{\mathrm{dm}} \sim 4-16 \times 10^{10} M_{\odot}\right)$. All of them 

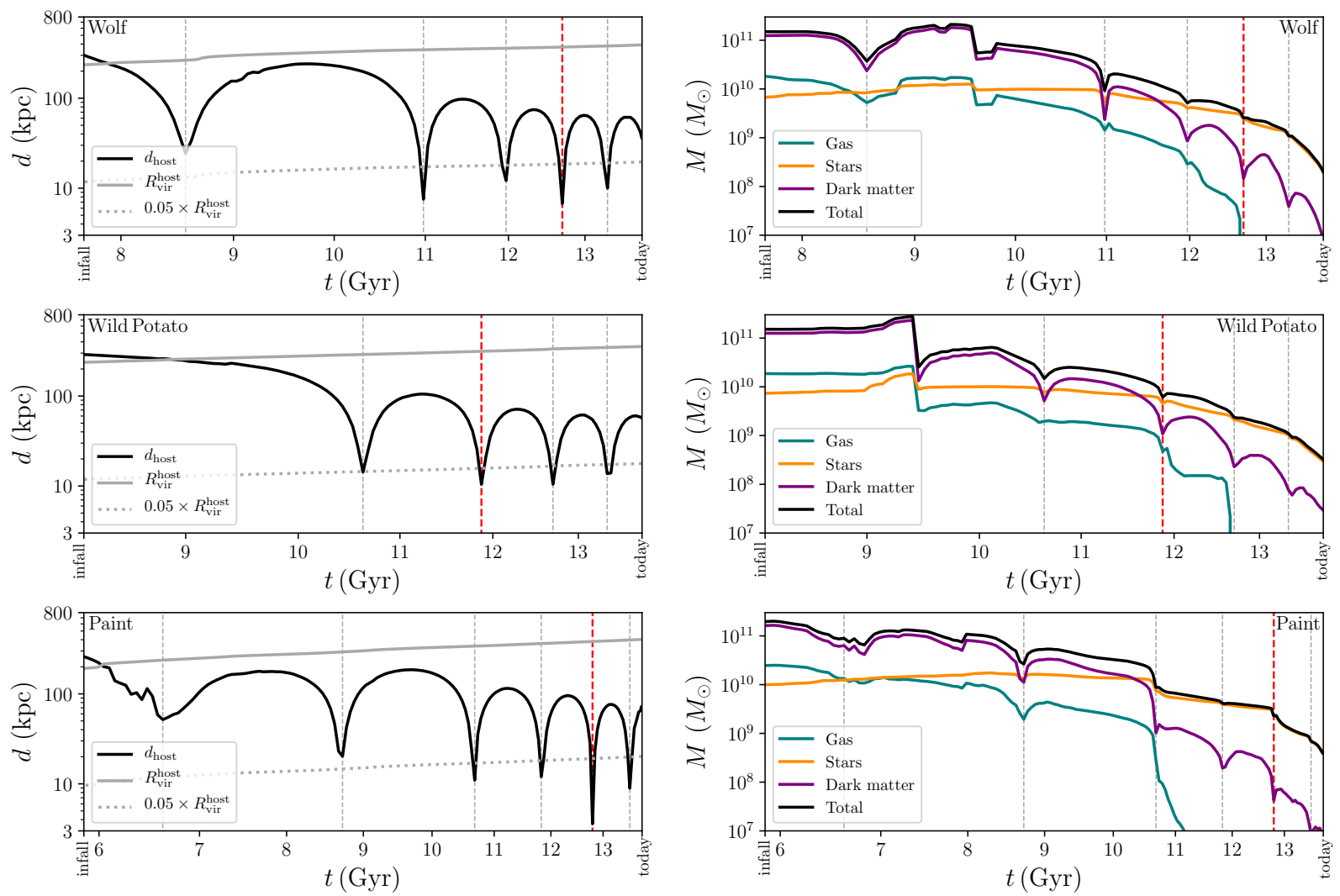

Figure 7a: The evolution of the seven dark-matter deficient galaxies. Left panels: Halo-centric distance (in physical units) versus cosmic time. The solid and dotted gray curves represents the virial radius of the host and 5\% thereof. Right panels: Mass (within the subhalo radius) versus time. The teal, orange and purple curves indicate mass in gas, stars and dark matter. The black curve represents total mass. The time range extends from $t_{\text {infall }}$, the infall time when the galaxy became a satellite for the first time, to the present time $(13.8 \mathrm{Gyr})$. The vertical gray lines indicate pericentric passages. The vertical red line highlights the time of the minimum halo-centric distance. 

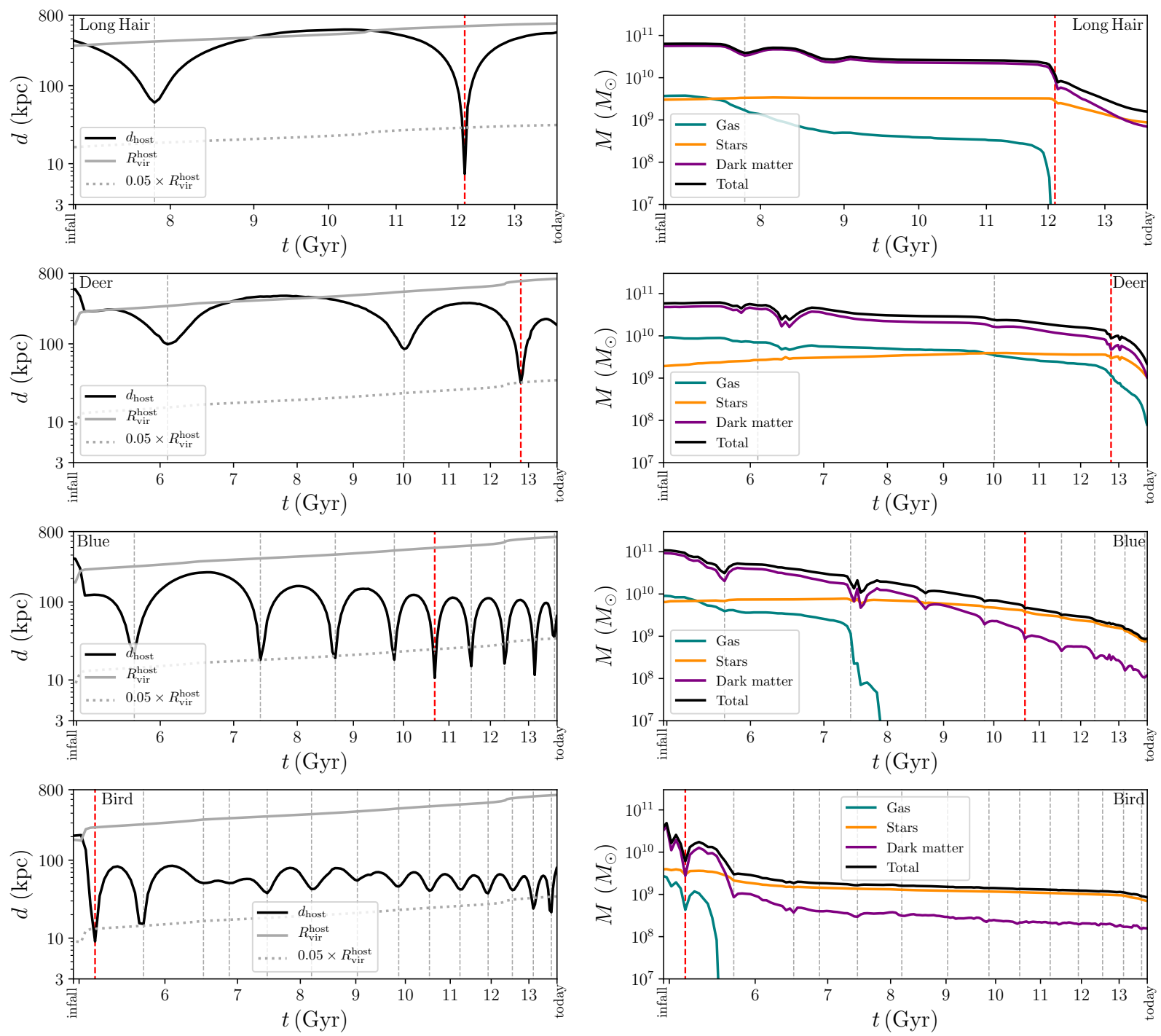

Figure 7b: Continuation of Figure 7a. 


\begin{tabular}{||c||ccccccc||}
\hline & Bird & Blue & Deer & LH & Paint & WP & Wolf \\
\hline \hline$M_{\star}^{z=0}$ & 7.0 & 7.6 & 10.0 & 8.8 & 3.9 & 3.0 & 2.0 \\
\hline$M_{\star}^{z=z_{\text {infall }}}$ & 39.4 & 64.5 & 19.5 & 30.2 & 98.5 & 73.4 & 68.8 \\
\hline$M_{\text {gas }}^{z=0}$ & 0.0 & 0.0 & 0.8 & 0.0 & 0.0 & 0.0 & 0.0 \\
\hline$M_{\text {gas }}^{z=z_{\text {in }}}$ & 26.7 & 89.7 & 91.8 & 36.8 & 247.2 & 187.3 & 176.5 \\
\hline$M_{\text {dm }}^{z=0}$ & 1.6 & 1.2 & 10.0 & 6.9 & 0.08 & 0.3 & 0.08 \\
\hline$M_{\mathrm{dm}}^{z=z_{\text {infall }}}$ & 353.2 & 920.0 & 480.8 & 565.9 & $1,622.6$ & $1,252.6$ & $1,252.9$ \\
\hline$f_{\star}^{\text {lost }}$ & $82.36 \%$ & $88.21 \%$ & $45.40 \%$ & $70.99 \%$ & $96.08 \%$ & $95.94 \%$ & $97.17 \%$ \\
\hline$f_{\text {gas }}^{\text {lost }}$ & $100 \%$ & $100 \%$ & $99.15 \%$ & $100 \%$ & $100 \%$ & $100 \%$ & $100 \%$ \\
\hline$f_{\text {dm }}^{\text {lost }}$ & $99.56 \%$ & $99.87 \%$ & $97.85 \%$ & $98.78 \%$ & $99.99 \%$ & $99.98 \%$ & $99.64 \%$ \\
\hline$z_{\text {infall }}$ & 1.26 & 1.23 & 1.23 & 0.76 & 1.00 & 0.56 & 0.63 \\
\hline
\end{tabular}

Table 2: Mass evolution of our seven dark-matter deficient galaxies. 'LH' and 'WP' denote for Long Hair and Wild Potato and $z_{\text {infall }}$ denotes the infall redshift. We display masses in units of $10^{8} M_{\odot}$ to emphasize changes. Quantities are within $R_{\text {vir }}$ (or $R_{\text {subhalo }}$ after infall) unless stated otherwise. (i) Stellar mass at $z=0$; (ii) stellar mass at infall; (iii) gas mass at $z=0$; (iv) gas mass at infall; (v) dark matter mass at $z=0$; (vi) dark matter mass at infall; (vii) fraction of stellar mass lost since infall; (viii) fraction of gas mass lost since infall; (ix) fraction of dark matter mass lost since infall; (x) infall redshift. 
lost over $99 \%$ of their dark-matter content between infall and the present time. See Table 2 for numerical values. We plan to explore the evolution of this set of seven galaxies in more detail in the future.

Simulations by other groups. The identification of galaxies lacking dark matter in cosmological simulations has been a challenge ${ }^{12-14,17-20}$. Whilst many of these studies have shown that galaxies can be produced with dark matter fractions lower than typical, the great majority of them do not produce galaxies below the $1: 1$ line on the $M_{\mathrm{dm}}-M_{\star}$ plane (Figure 2). The sole exception is Ref. ${ }^{13}$, who find one single low-mass object in an intermediate-resolution simulation meeting the above requirement (this object has $M_{\mathrm{dm}} / M_{\star} \sim 40 \%$, comparable to Deer). We note that their low-resolution samples do not separate low- and intermediate-mass galaxy populations, making comparisons with our work impossible. Moreover, none of these works report simulated galaxies with internal properties (i.e., 1D line-of-sight velocity dispersions, galactic sizes and Sérsic indices) in agreement with observations (Figure 3 and Table 1). Lastly, we predict the existence of dark-matter deficient galaxies with extremely low values of $M_{\mathrm{dm}} / M_{\star}$ : three below $10 \%$ and one below $1 \%$. Future observations and improved methodologies for measuring $M_{\mathrm{dm}} / M_{\star}$, will help confirm or refute these predictions.

There are several possibilities that could explain why results similar to ours have not been reported in past simulations. First, as mentioned in the main text, our simulations are relatively unique in that they explicitly track dense, molecular gas in low-mass galaxies within a volume that is large enough to contain several massive group-size halos. There are published simulations ${ }^{19,21}$ with similar formal spatial resolution to our own within comparable cosmological volumes, but 
these utilize different star-formation and galaxy-formation physics. In particular, our star-formation threshold is $>30$ times higher than used in these comparably-sized simulations and this allows us to track the internal baryonic structure of galaxies with higher precision. Another possibility is that some simulations do in fact produce such extreme objects, but their automated galaxy-recovery algorithms might pose an impediment to their query (recall that we employ a visual-recovery technique, which may not be feasible for larger runs).

Alternatively, many authors employ idealized (non-cosmological) simulations ${ }^{5-7,11}$. These controlled numerical experiments offer unique insight on the role of satellite-host interactions at resolution levels often inaccessible to cosmological simulations. Major caveats in this approach include the need to make assumptions about the initial structure of the satellite's progenitors, the host and the orbital geometry of the satellite-central interaction (but see Ref. ${ }^{8}$, who circumvents the first caveat). Another limitation is that this technique operates under the presumption that one specific mechanism must be responsible for the creation of dark-matter deficient galaxies. Our work therefore validates this scenario in a more natural, cosmologically-motivated fashion. Namely, that close satellite-host encounters drive the formation of dark-matter deficient galaxies.

Data availability. The datasets generated during and/or analysed during the current study are available from the corresponding author on reasonable request.

Code availability. The G I ZMO hydro-solver publicly available at https://bitbucket.org/phopkins/gizmopublic/src/master/. The Amiga Halo Finder is publicly available at http://popia.ft.uam.es/AHF/Download.html. yt is publicly available at https://yt-project.org/. FIRE 
Studio is publicly available at https://github.com/agurvich/FIRE_studio. The Python scripts used to create the figures in the current study are available from the corresponding author on reasonable request.

1. Knollmann, S. R. \& Knebe, A. AHF: Amiga's Halo Finder. ApJSS 182, 2, 608-624 (2009).

2. Knebe, A. et al. Haloes gone MAD: The Halo-Finder Comparison Project. MNRAS 415, 3, 2293-2318 (2011).

3. Turk, M. J. et al. yt: A Multi-code Analysis Toolkit for Astrophysical Simulation Data. ApJS 192, 1, 9, 16 (2011).

4. Elmegreen, B. G., Kaufman, M. \& Thomasson, M. An Interaction Model for the Formation of Dwarf Galaxies and 108 Msun Clouds in Spiral Disks AJ 412, 90, (1993).

5. Ogiya, G. Tidal stripping as a possible origin of the ultra diffuse galaxy lacking dark matter. MNRAS 480, L106-L110 (2018).

6. Huo, R. Refracting into ultra-diffuse galaxy NGC 1052-DF2 by passing near the centre of NGC 1052. MNRAS 495, L144-L148 (2020).

7. Nusser, A. A Scenario for Ultradiffuse Satellite Galaxies with Low Velocity Dispersions: The Case of [KKS 2000]04. ApJ 893, 66, 11 (2020).

8. Macciò, A. V., et al. Creating a galaxy lacking dark matter in a dark matter-dominated universe. MNRAS 501, 1, 693-700 (2021). 
Acknowledgements JM thanks Pieter van Dokkum, Janice Hudgings, Dwight Whitaker, David Tanenbaum and Robert Gaines for comments on an earlier draft, and Christopher Hayward for data-transfer support. SD is supported by NASA through Hubble Fellowship grant HST-HF2-51454.001-A awarded by the Space Telescope Science Institute, which is operated by the Association of Universities for Research in Astronomy, Incorporated, under NASA contract NAS5-26555. JSB, FJM and SY were supported by NSF grant AST-1910346. RF acknowledges financial support from the Swiss National Science Foundation (grant no PP00P2_157591, PP00P2_194814 and 200021_188552). Support for PFH was provided by NSF Research Grants 1911233 \& 20009234, NSF CAREER grant 1455342, NASA grants 80NSSC18K0562, HST-AR-15800.001-A. AL was supported by NASA grant 80NSSSC20K1469. ZH is supported by a Gary McCue postdoctoral fellowship through the Center for Cosmology at UC Irvine. CK was supported by a National Science Foundation Graduate Research Fellowship Program under grant DGE-1839285. CAFG was supported by NSF through grants AST-1715216 and CAREER award AST-1652522; by NASA through grant 17-ATP17-0067; by STScI through grant HST-AR-16124.001-A; and by the Research Corporation for Science Advancement through a Cottrell Scholar Award and a Scialog Award. AW received support from NSF CAREER grant 2045928; NASA ATP grants 80NSSC18K1097 and 80NSSC20K0513; HST grants AR-15809 and GO-15902 from STScI; a Scialog Award from the Heising-Simons Foundation; and a Hellman Fellowship. DAA acknowledges support by NSF grant AST-2009687 and by the Flatiron Institute, which is supported by the Simons Foundation. MBK acknowledges support from NSF CAREER award AST-1752913, NSF grant AST-1910346, NASA grant NNX17AG29G and HST-AR-15006, HSTAR-15809, HST-GO-15658, HST-GO-15901, HST-GO-15902, HST-AR-16159 and HST-GO-16226 from STScI. EQ was supported in part by a Simons Investigator Award from the Simons Foundation and by NSF grant AST-1715070. DK was supported by NSF grant AST-1715101. Numerical calculations were run on the Caltech compute cluster "Wheeler," allocations FTA-HopkinsAST20016 supported by the NSF and 
TACC and NASA HEC SMD-16-7592. We acknowledge PRACE for awarding us access to MareNostrum at Barcelona Supercomputing Center (BSC), Spain. This research was partly carried out via the Frontera computing project at the Texas Advanced Computing Center. Frontera is made possible by National Science Foundation award OAC-1818253. This work was supported in part by a grant from the Swiss National Supercomputing Centre (CSCS) under project IDs s697 and s698. We acknowledge access to Piz Daint at the Swiss National Supercomputing Centre, Switzerland under the University of Zurich's share with the project ID uzh18. Additional computing support was provided by S3IT resources at the University of Zurich. JM (an astronomer of Indigenous ancestry, non-Cherokee) thanks Doug Ingram (a Cherokee physicist) for sharing his knowledge about the Cherokee Nation and his ideas to avoid cultural appropriation. This work was conducted on Tongva-Gabrielino land.

Author Contributions. J.M. conducted the analysis and designed the manuscript, with substantial input from S.D. and J.S.B. S.D collected observational data from the literature and verified the theory-observations comparisons. R.F. ran the cosmological simulation used in this work. O.C. generated the halo catalogs and merger trees. A.G. provided support for the creation of mock images. A.L. and C.B.H. provided support to interface with the particle data. C.K. calculated $g$-band effective radii and Sérsic indices. All authors contributed to the final creation of the manuscript and figures.

Competing Interests. The authors declare that they have no competing financial interests.

Correspondence. Correspondence and requests for materials should be addressed to

J.M. (email: jorge.moreno@pomona.edu). 